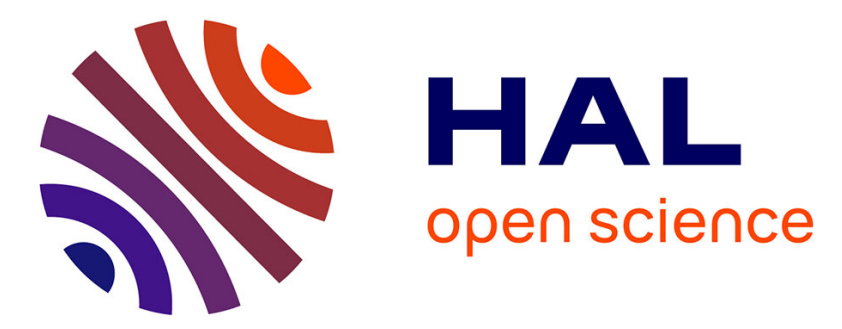

\title{
FRACTURE MECHANICS STUDY OF CERAMIC-CERAMIC COMPOSITES
}

D. Rouby, M. Loumba-Chaze, Gilbert Fantozzi, J. Tron

\section{To cite this version:}

D. Rouby, M. Loumba-Chaze, Gilbert Fantozzi, J. Tron. FRACTURE MECHANICS STUDY OF CERAMIC-CERAMIC COMPOSITES. Journal de Physique Colloques, 1986, 47 (C1), pp.C1-613C1-616. 10.1051/jphyscol:1986193 . jpa-00225624

\section{HAL Id: jpa-00225624 https://hal.science/jpa-00225624}

Submitted on 1 Jan 1986

HAL is a multi-disciplinary open access archive for the deposit and dissemination of scientific research documents, whether they are published or not. The documents may come from teaching and research institutions in France or abroad, or from public or private research centers.
L'archive ouverte pluridisciplinaire HAL, est destinée au dépôt et à la diffusion de documents scientifiques de niveau recherche, publiés ou non, émanant des établissements d'enseignement et de recherche français ou étrangers, des laboratoires publics ou privés. 


\title{
FRACTURE MECHANICS STUDY OF CERAMIC-CERAMIC COMPOSITES
}

\author{
D. ROUBY, M. LOUMBA-CHAZE, G. FANTOZZI and J. TRON* \\ I.N.S.A. de Lyon, G.E.M.P.P.M.,UA 341, Bât. 502, \\ F-69621 Villeurbanne Cedex, France \\ *Société Aérospatiale-Aquitaine, B.P. 11 , \\ F-33160 st Médard en Jalles, France
}

\begin{abstract}
Résumé - Ies matériaux étudiés sont des composites à matrice silice renforcée par des fibres courtes de Nextel ou de SiC distribuées aléatoirement. Les essais de détermination des courbes de résistance à la propagation de la fissure sont effectués sur des éprouvettes de flexion entaillées (SENB) et analysés dans le cas d'un comportement non linéaire (Courbes $\mathrm{J}_{\mathrm{R}}$ )
\end{abstract}

Abstract - The materials used in this study are silica matrix composites reinforced with short random Nextel or SiC Eibers. The tests to determine the crack propagation resistance curves are performed on SENB specimens and analysed in the case of non linear behaviour ( $J_{R}$ curves).

\section{I - INTRODUCTTON}

Dans les matériaux composites à matrice fragile, la multiplicité et la complexité des mécanismes microscopiques associés à la propagation d'une fissure ne permet pas de caractér iser le comportement à la rupture par un seul paramètre critique comne $\mathrm{K}_{\text {Ic }}$ ou $\mathrm{G}_{\text {Ic }}$ pour les matériaux parfaitement fragiles et linéaires. Aussi, nous avons déterminé les courbes de résistance à la propagation d'une fissure dans le cas d'un comportement non linéaire et pour deux types de matériaux composites à fibres courtes aléatoires et à matrice silice. Ces matériaux ont été élaborés dans des conditions données ; les résultats obtenus ne doivent donc pas être considérés conme caractéristiques de cette classe de matériaux du fait de la très grande influence des conditions d'élaboration sur leur comportement mécanique.

\section{II - METHODE EXPERIMENTALE}

Les matériaux étudiés, élaborés par la Société Nationale Industrielle Aérospatiale sont des composites à matrice silice renforcée par des fibres courtes de Nextel $312^{\mathrm{R}}$ (produit par 3M; $\mathrm{Al}_{2} \mathrm{O}_{3} 62 \%, \mathrm{~B}_{2} \mathrm{O}_{3} 148, \mathrm{SiO}_{2} 248$; diametre moyen: $11 \mu \mathrm{m}$; densité: 2.7 $\mathrm{g} / \mathrm{cm}^{3}$; résistance: $1720 \mathrm{MPa}$ ) et $\mathrm{SiC}$ (Nicalon $\mathrm{R}$, procuit par Nippon Carbon; SiC 64\%, C $15 \%, \mathrm{SiO}_{2} 21 \%$; diametre: $13-15 \mu \mathrm{m}$; densité: $2.55 \mathrm{~g} / \mathrm{cm}^{3}$; résitance: $2450-2900 \mathrm{MPa}$ ), densifiés par voie liquide (barbotine Ludox) et frittages successifs. Les fibres, broyées, ont une longueur inférieure à $500 \mu \mathrm{m}$, elles ne forment pas d'amas et elles présentent une orientation préférentielle parallèlement au plan des plaques ainsi élaborées. La porosité des matériaux testés est de I'ordre de $30 \%$.

Les éprouvettes de dimensions $4 \times 4 \times 70 \mathrm{~mm}^{3}$ sont prélevées dans les plaques. L'entaille est réalisée, au milieu du barreau, au disque diamant (extrémité arrondie, rayon : $0.15 \mathrm{~mm})$; le fond de l'entaille est orienté perpendiculairement au 


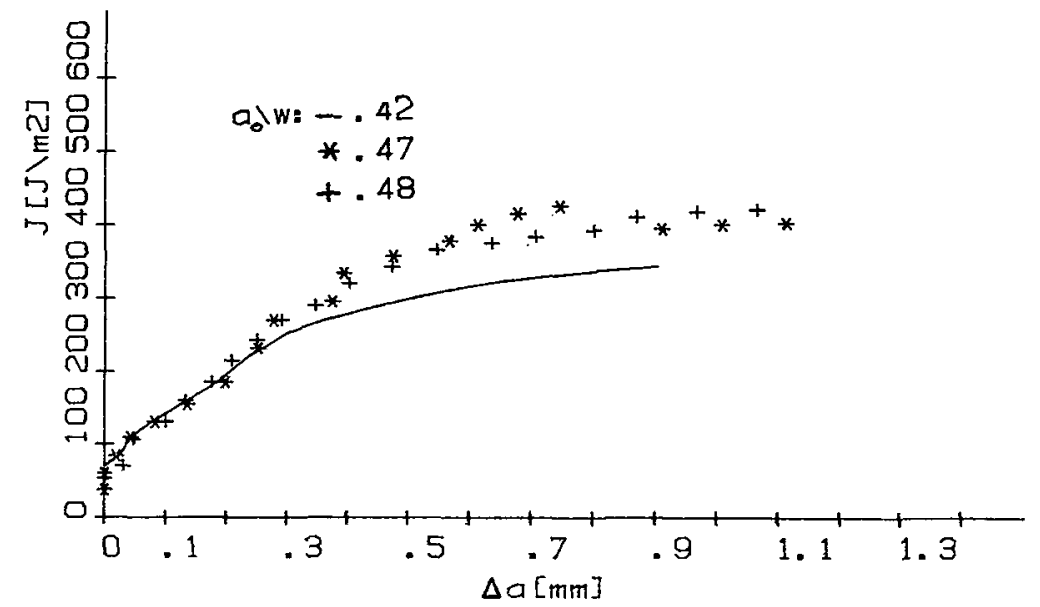

Fig. 1 : Fibres Nextel, $v_{E}=208$. Courbes $J_{R}$. Nextel fibers, $v_{f}=208$. J J curves.

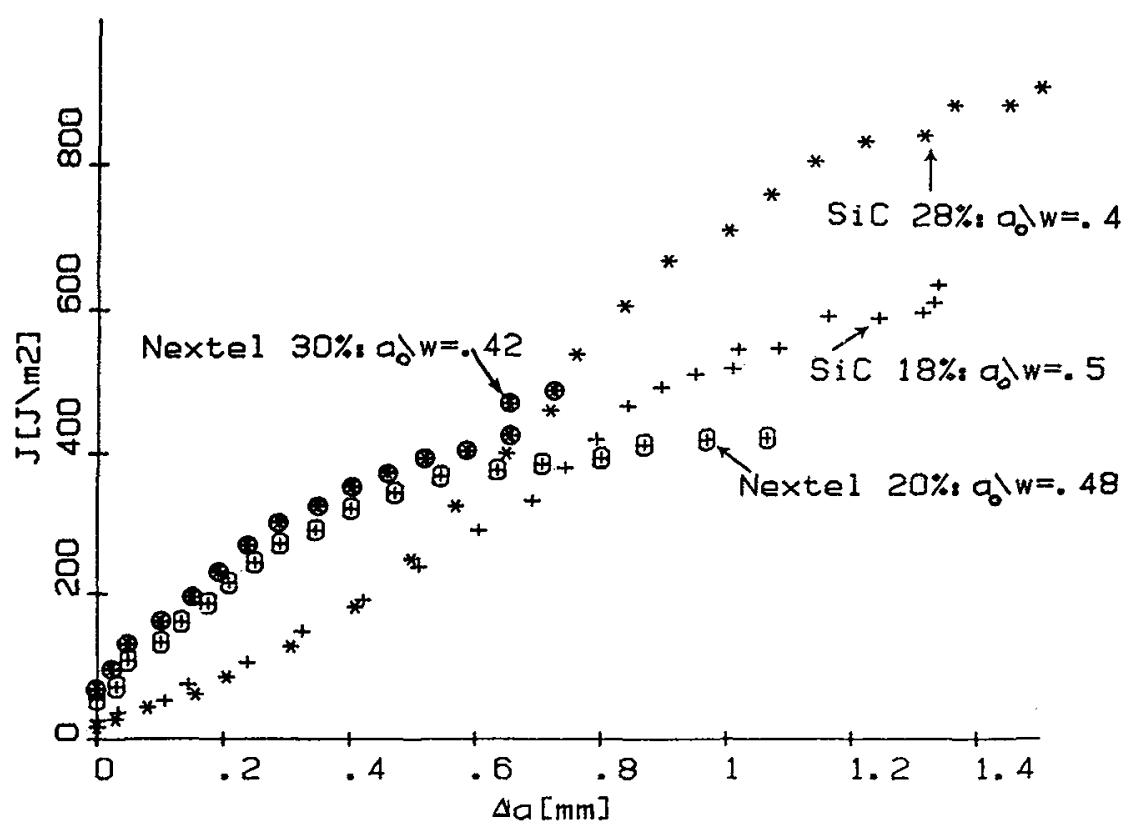

Fig. 2 : Courbes $J_{R}$. Le renfort, $v_{E}$ et $a_{0} / w$ sont spécifiés. $\mathrm{J}_{\mathrm{R}}$ curves. Fibers, $\mathrm{v}_{f}$ and $a_{o} / w$ are given. 
plan de la plaque initiale. Les essais ont été effectués en flexion trois points sur une machine INSTRON, modèle 1195 équipée d'une cellule de capacité $5 \mathrm{KN}$. La vitesse du point d'application de la charge est fixée à $0,2 \mathrm{~mm} / \mathrm{m}$.

Pour suivre la propagation de la fissure, les éprouvettes subissent des cycles successifs de charge-décharge et la compliance est mesurée à chaque cycle. La profondeur de la fissure effective est déterminée par l'expression itérative suivante $/ 1 /$ :

$$
a_{n+1}=a_{n}+\frac{w-a_{n}}{2} \cdot \frac{c_{n+1}-c_{n}}{c_{n+1}}
$$

avec: $a_{n}, c_{n}$ : respectivement profondeur de fissure et compliance après le cycle $n$; $W-a_{n}$ : ligament de matière; $W$ : hauteur de l'éprouvette.

Les courbes de résistance à la propagation ont été déterminées dans l'hypothèse non linéaire, à partir du concept de 1 'intégrale $\mathrm{J} / 2 \%$. Pour déterminer la valeur de $\mathrm{J}$ après chaque cycle et donc en fonction de l'accroissenent de fissure $\Delta a$ (courbe $J_{R}$ ), on utilise 1 'expression suivante, proposée par Garwood et al /3/ :

$$
J_{n+1}=J_{n} \frac{W-a_{n+1}}{W-a_{n}}+\frac{2 U_{n}}{B\left(w-a_{n}\right)} \quad \text { et } \quad J_{0}=\frac{2 U_{0}}{B\left(w-a_{0}\right)}
$$

avec: $U_{n}$ : énergie nécessaire au système pour faire avancer la fissure de $a_{n}-a_{n-1}$ et $J_{\mathrm{O}}$ : énergie d'initiation de la fissuration. $\Delta a=a_{n}-a_{O}$.

\section{III - RESULTATS ET DISCUSSION}

Le comportement du matériau n'est pas élastique en effet après décharge, 1 'éprouvette présente une flèche résiduelle. Ceci reflète un comportement pseudo-plastique et dans ces conditions les valeurs de J données par I'équ. (2) prennent en compte l'énergie absorbée par la déformation permanente $/ 4 /$.

Un résultat typique concernant le matériau Nextel-Silice est donné fig. 1, pour une fraction volumique de fibres nominales $v_{f}=208$; pour des taux de fibres supérieurs $(25$ et 30 \%) le comportement est tout-à-fait comparable. On observe globalement une énergie a'initiation $\mathrm{J}_{0}$ comprise entre 50 et $70 \mathrm{~J} / \mathrm{m}^{2} ; \mathrm{J}_{0}$ reflète le début de l'extension de la zone endommagée en fond d'entaille dans laquelle ont lieu des fissurations de la matrice et des décollements fibre-matrice. J croît ensuite pour atteinare un plateau $J_{\max }$ dont le niveau se situe entre 400 et $500 \mathrm{~J} / \mathrm{m}^{2}$. L'augmentation de $J$ avec a est due à la croissance de la zone endommagée et en particulier au mécanisme d'extraction de fibres (pul1-out) lorsque la fissure progresse dans la matrice. L'énergie absorbée par ce dernier mécanisme est proportionnelle à $v_{f}$, à la proportion de fibres ayant été extraites et à la longueur moyenne extraite ; elle est aussi d'autant plus grande que la contrainte critique de glissement à l'interface est faible $/ 5 \%$

La fig. 2 montre la comparaison entre le renfort Nextel et Sic. On constate qu'aux faibles $\Delta a, J(S i C)$ est inférieur à $J$ (Nextel) et la tendance est inversée aux forts accroissements : $\mathrm{J}_{\max }(\mathrm{SiC})<\mathrm{J}_{\max }$ (Nextel). Ce résultat semble indiquer que le mécanisme de pull-out est plus important dans le cas du renfort SiC. On observe en effet, sur le faciès de rupture (fig. 4), un plus grand nombre de fibres extraites et de plus grande longueur que dans le cas du renfort Nextel (Fig. 3) où une forte proportion de fibres sont rompues dans le plan de fissure. Dans le cas du renfort Nextel, 1'interface est résistant et ce matériau est meilleur pour l'initiation alors que dans le cas du renfort SiC, I'interface est plus faible et ce matériau est plus résistant à la propagation. 


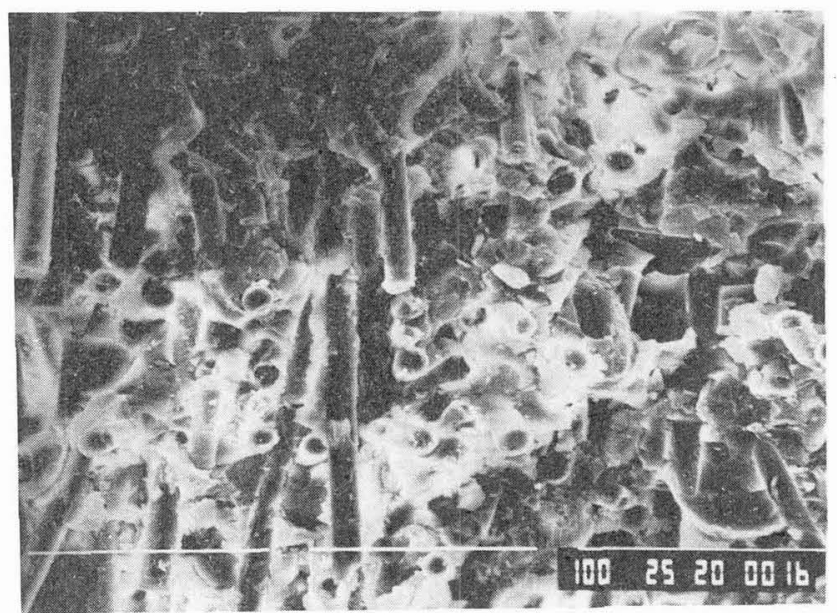

Fig. 3 : Fibres Nextel, $v_{f}=258$. Fractographie $(x 300)$. Nextel fibers: $v_{f}=25 \%$. SEM fractography $(x 300)$.

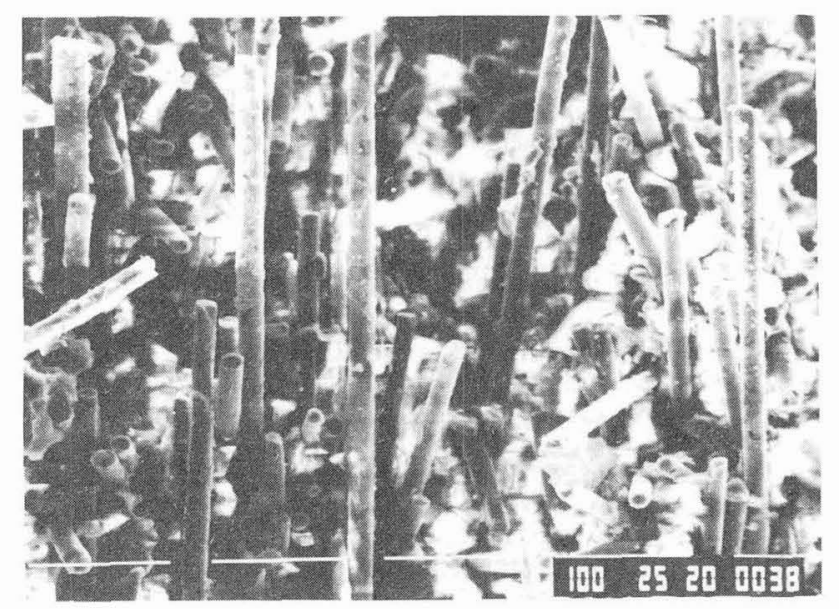

Fig. 4 : Fibres SiC, $v_{f}=288$. Fractographie $(x 200)$. Sic fibers, $v_{f}=28 \%$. SEM fractography $(x 200)$.

\section{REFERENCES}

1/ Data H., Paris P. et Irwin G., The stress Analysis Handbook (1973).

/2/ Rice J.R., J. Appl. Mech. 35 (1968) 379.

/3/ Garwood S., Robinson J., Turner C., Int. J. Fract. II (1975) 528.

/4/ Ferry F., Thèse Docteur-Ingénieur (1984), Lyon.

15/ Jayatilaka A.S., Fracture of Engineering brittle materials (1979) Applied Science Pub., London. 